\section{EFECTIVIDAD DE LAS INTERVENCIONES COGNITIVAS EN LA PREVENCIÓN DEL DETERIORO DE LA MEMORIA EN LAS PERSONAS MAYORES SANAS}

Novoa, Ana M., Juarez Olga y Nebot Manel Ref: Gaceta Sanitaria, Set.-Oct.2008,Vol.22, No5, p.474-842, ISSN 0213-9111

En los últimos años han aparecido diversas intervenciones no farmacológicas destinadas a prevenir el deterioro cognitivo asociado a la edad, concretamente los problemas de memoria, cuya efectividad no ha sido suficientemente evaluada.

El objetivo de este trabajo es establecer la efectividad de las intervenciones cognitivas en la prevención del deterioro de la memoria asociado a la edad mediante la revisión de la literatura científica.

Se realizó una búsqueda exhaustiva en PubMed. Se incluyeron los estudios publicados desde 1990 que evaluaran la efectividad de una intervención cognitiva sobre la memoria en personas mayores sin deterioro cognitivo leve ni demencia, y se clasificaron según su calidad. Se evaluaron los efectos a corto y largo plazo, sobre el rendimiento en tareas de memoria objetiva y subjetiva y la transferencia a otros dominios cognitivos.

En los resultados se deja constancia que de los 25 estudios que cumplieron los criterios de inclusión, XI (44\%) fueron considerados de elevada calidad, de los cuales todos, excepto uno mejoraron algún indicador de memoria. El tipo de memoria más evaluada fue la memoria objetiva reciente verbal, que mejoró en 8 de 9 estudios de elevada calidad. La memoria objetiva reciente no verbal y de asociación, evaluadas principalmente por los estudios de calidad media, mejoraron en 4 de 7 , y en 10 de 11 estudios, respectivamente. Los resultados referentes a memoria subjetiva fueron muy heterogéneos. La transferencia únicamente se observó en uno de 7 estudios de elevada calidad.

Se concluye que los resultados sugieren que determinadas intervenciones cognitivas desarrolladas en personas mayores sanas son efectivas en la prevención del deterioro de la memoria, especialmente sobre la memoria reciente verbal.

Palabras clave: Salud mental, memoria, evaluación de programas, ancianos, prevención.

\section{IMPACTO DEL CUIDADO INFORMAL EN LA SALUD Y CALIDAD DE VIDA DE LAS PERSONAS CUIDADORAS: ANÁLISIS DE LAS DESIGUALDADES DE GÉNERO}

Larrañaga Isabel, Martín Unal, Bacigalupe Amaia, et al.

Ref: Gaceta Sanitaria, Sept-Oct. 2008, Vol. 22, No5,p.443-450. ISSN 0213-911

Los objetivos planteados en el presente trabajo son: caracterizar a los cuidadores y cuidadoras informales, cuantificar el efecto que la actividad de cuidar tiene sobre su salud y determinar si éste es diferente según sexo.

Se trata de un estudio descriptivo basado en la Encuesta de Servicios de Salud del País Vasco 2002. Se compararon la salud física y mental, la utilización de servicios, la salud social y la calidad de vida relacionada con la salud ( CVRS) en personas cuidadoras $(n=836)$ y no cuidadoras $(n=5.706)$. Se calcularon los modelos de regresión logística para estimar la asociación entre el cuidado y los resultados en salud por sexos, ajustados por edad, nivel socioeconómico y situación laboral.

En cuanto a los resultados, las cuidadoras puntuaron peor que las no cuidadoras en 6 de los 10 indicadores, y los cuidadores en 4. Las asociaciones entre el cuidado (cuidar frente a no cuidar) y los resultados en salud fueron más notorias en las mujeres, excepto en la salud social. Al considerar la intensidad de la carga del cuidado, los hombres cuidadores con carga elevada presentaron riesgos iguales o superiores de mala CVRS (física hombres: odds ratio $(\mathrm{OR})=3.0$ intervalo de confianza del 95\% ( IC 95\%): 1,4-4,3; mental mujeres: $\mathrm{OR}=2,5$ IC95\%:1,7-3,7) y poco apoyo social ( apoyo afectivo hombres: OR=2,6, IC95\%:1,5- 
4,6: apoyo afectivo mujeres: $\mathrm{OR}=1,5 \mathrm{IC} 95 \%$ : 1,0 - 2,3). Sin embargo, las cuidadoras mostraron mayor riesgo de sedentarismo, falta de sueño, número de consultas y peor salud mental.

Se concluye que cuidar afecta negativamente a la salud de quienes cuidan, pero entraña mayores riesgos para las mujeres por la mayor carga de trabajo que deben asumir. Cuando los hombres aumentan la carga de cuidador, las desigualdades de género se reducen o invierten.

Palabras clave: Cuidado informal; desigualdades de género; desigualdades en salud; apoyo social; calidad de vida relacionada con la salud.

\section{CHANGE IN RECIPROCITY AS A PREDICTOR OF DEPRESSIVE SYMPTOMS: A PROSPECTIVE COHORTE STUDY OF FINNISH WOMEN AND MEN.}

Ari Väänänen, Abraham P Buunk, Mika Kivimäki, Jussi Vahtera, Markku Koskenvuo

Rev: Social Science \& Medicine 67 (2008) 1907-1916

El propósito del estudio fue examinar las diferencias de género en la asociación entre cambios en la ecuación dar / recibir respecto a las relaciones personales cercanas y los síntomas depresivos. Los datos estudiados provienen de una cohorte a 5 años en Finlandia (HeSSup Study, N=18,445). Luego de ajustar por características demográficas, eventos vitales negativos recientes, línea base de síntomas depresivos, hostilidad y apoyo de redes sociales, un cambio del balance hacia recibir apoyo resultó un factor de riesgo significativo para futuros síntomas depresivos entre las mujeres. Al contrario, hombres cuyo balance dar / recibir se había desplazado hacia dar mostraron un mayor riesgo de futuros síntomas depresivos que otros hombres. Cuando los análisis fueron replicados en una sub-cohorte de participantes inicialmente no deprimidos que vivían relaciones recíprocas y no habían tenido eventos vitales recientes, los resultados se hicieron aún más pronunciados entre las mujeres, aunque no entre los hombres. Se concluye que, para las mujeres, un cambio en sus relaciones cercanas hacia recibir apoyo puede conducir a un riesgo aumentado de síntomas depresivos, mientras para los hombres un cambio hacia el dar puede tener un impacto paralelo, aunque menos marcado.

Palabras Clave: relaciones personales cercanas, síntomas depresivos. 